\title{
High-Resolution Full-Field X-ray Microscope for 20-keV X-rays with Multilayer Imaging Mirrors
}

\author{
$\underline{\text { Satoshi Matsuyama }}^{1, *}$, Jumpei Yamada ${ }^{1}$, Kentaro Hata $^{1}$, Yoshiki Kohmura ${ }^{2}$, Makina Yabashi ${ }^{2}$, \\ Tetsuya Ishikawa ${ }^{2}$, and Kazuto Yamauchi ${ }^{1}$ \\ ${ }^{1}$ Department of Precision Science and Technology, Graduate School of Engineering, Osaka University, \\ 2-1 Yamada-oka, Suita, Osaka 565-0871, Japan \\ ${ }^{2}$ SPring-8/RIKEN, 1-1-1 Kouto, Sayo, Hyogo 679-5198, Japan \\ * Corresponding author, matsuyama@ prec.eng.osaka-u.ac.jp
}

Full-field X-ray microscopes are extremely powerful and, in contrast to scanning-type X-ray microscopes and coherent diffraction imaging, capable of imaging objects without the need for sample scanning and complex data analysis. Thus, they are compatible with real-time observation and spectronanoscopy. The performance of this type of microscope is strongly dependent on the characteristics and quality of the imaging optics used. Fresnel zone plates (FZPs), the most important imaging optics at the X-ray region, enable high-resolution imaging. However, general FZPs are barely capable of focusing/imaging X-rays with energy greater than $15 \mathrm{keV}$ since the diffraction efficiency tends to decrease with increase in the X-ray energy. High-energy X-rays, though, are extremely useful for imaging the interior of thick samples, such as semiconductor devices and complex materials. One imaging optics compatible with such $\mathrm{X}$-rays is a multilayer imaging mirror. Out of the available X-ray mirrors, advanced Kirkpatrick-Baez mirror $(\mathrm{AKB})$ optics $^{[1]}$ is a realistic solution for high resolution because of the possibility of mirror fabrication with a shape accuracy of a few nanometers. In this work, we have developed and demonstrated AKB optics comprising four multilayer mirrors (Fig. 1).

An AKB optics with multilayer mirrors for medium-range-energy (approximately $20 \mathrm{keV}$ ) X-rays were developed by modifying a total-reflection AKB optics that was previously fabricated by our group ${ }^{[1]}$. The each imaging mirror comprises concave hyperbolic and concave elliptical parts following Wolter type I optics rules. As reported previously, the residual figure error from the designed shape was $1 \mathrm{~nm}$, which is approximately equal to the tolerance required for obtaining ideal spatial resolution at $20 \mathrm{keV}$. Fig. 1 shows a schematic of the imaging optics. The numerical aperture, working distance, and magnification (vertical and horizontal) were $\sim 1.5 \times 10^{-3}, 35 \mathrm{~mm}, 25.8$, and 83.8, respectively. The four mirrors were covered with laterally graded multilayers of platinum and carbon using DC magnetron sputtering technique ${ }^{[2]}$. The minimum (maximum) period of the multilayer and total number of periods were $6.0(15.0) \mathrm{nm}$ and 10, respectively. The expected reflectivity after quadruple reflections was $22 \%$ at $20 \mathrm{keV}$. Fig. 2 shows the expected point spread function, which has a full width at half maximum of $18 \mathrm{~nm}$.

An X-ray imaging experiment was performed at the second experimental hutch of BL29XUL (SPring-8) (Fig. 1). 20-keV X-rays, monochromatized using a Si 111 double-crystal monochromator, were used. A diffuser was placed immediately upstream of the samples. A Siemens star chart (XRESO-20, NTT Advanced Technology Corporation), made of tantalum with a $20 \mathrm{~nm}$ feature at the innermost region and a thickness of $100 \mathrm{~nm}$, was observed. Tantalum with a thickness of $100 \mathrm{~nm}$ is absorbed to an extent of only $1 \%$ at $20 \mathrm{keV}$. The X-ray camera used has an effective field of view of $1.3 \times 1.3 \mathrm{~mm}^{2}$ and a pixel size of $650 \mathrm{~nm} /$ pixel, which correspond to $52 \times 16(\mathrm{~V} \times \mathrm{H}) \mu \mathrm{m}^{2}$ and $25 \times 8(\mathrm{~V} \times \mathrm{H}) \mathrm{nm} / \mathrm{pixel}$, respectively, on the object plane. Fig. 3 shows a recorded bright-field image. The data was processed by 
flat-field correction, in which a sample image is normalized using an empty image obtained under the same experimental conditions, but without the samples. Consequently, a $50 \mathrm{~nm}$ feature could be resolved, and a $40 \mathrm{~nm}$ feature was barely visible in the horizontal direction only. The fabricated microscope system was stable over a period of 1 day or even more, thanks to the monolithic structure on the imaging mirror between the elliptical and hyperbolic parts. This demonstration reveals the feasibility of developing a practical and high-resolution full-field microscope for X-rays with energy approximately equal to $20 \mathrm{keV}$. In the future, we plan to construct a full-field microscope system based on new AKB optics with concave and convex mirrors ${ }^{[3]}$.

\section{References:}

[1] S. Matsuyama et al., Sci. Rep. 7, 46358 (2017)

[2] J. Kim et al., Opt. Express 23, 29032 (2015)

[3] J. Yamada et al., Appl. Opt. 56, 967 (2017)

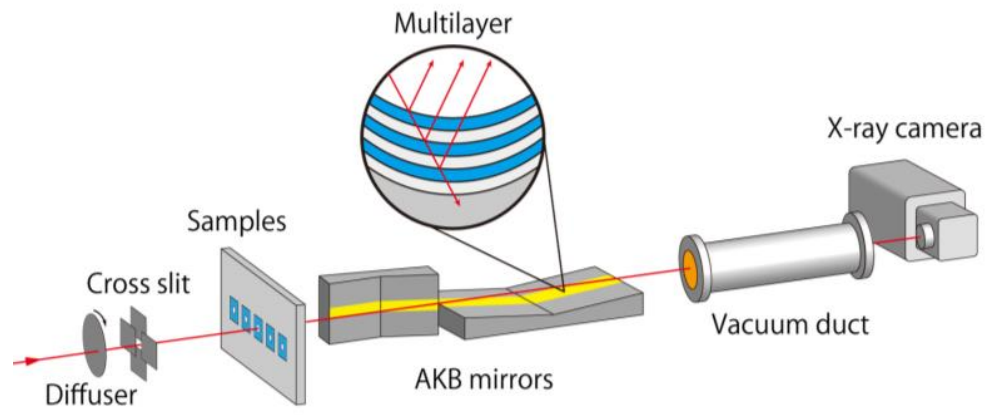

Fig. 1 Schematic of a full-field microscope with AKB optics.

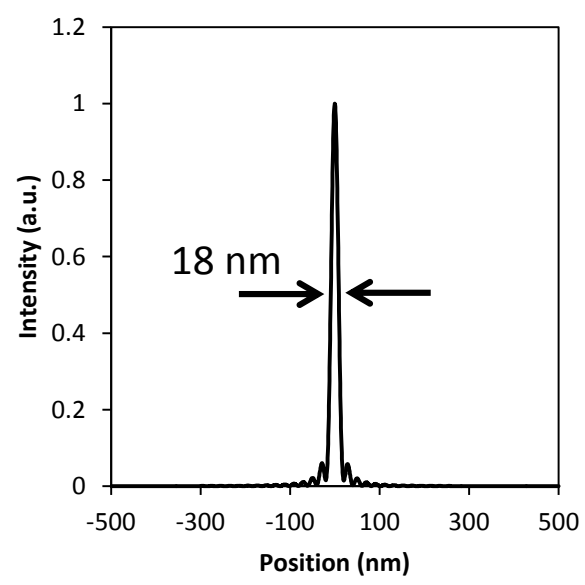

Fig. 2 Simulated point spread function of the imaging optics (horizontal direction).

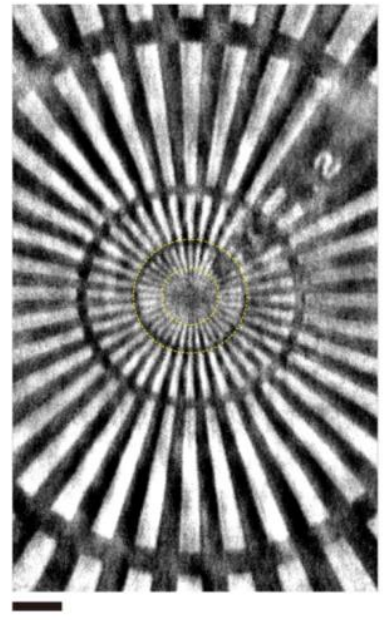

Fig. 3 Bright-field X-ray image of a tantalum Siemens star with a minimum feature $=20 \mathrm{~nm}$ and thickness $=100 \mathrm{~nm}$. X-ray energy $=20 \mathrm{keV}$. Exposure $=30 \mathrm{~s}$. Bar $=1 \mu \mathrm{m}$. The innermost yellow circle represents a $50 \mathrm{~nm}$ feature and the next concentric circle represents a $100 \mathrm{~nm}$ feature. 